\title{
Pragmatic language production deficits in Parkinson's disease
}

\author{
Thomas Holtgraves*, Kelly Fogle, Lindsay Marsh
}

Department of Psychological Science, Ball State University, Muncie, USA; *Corresponding Author: 00t0holtgrav@bsu.edu

Received 30 November 2012; revised 6 January 2013; accepted 16 january 2013

\begin{abstract}
This study examines pragmatic language production deficits in people with Parkinson's disease (PD). Participants (PD and non-PD) were interviewed and their responses coded for degree of informativeness. PD participants were more under-informative than non-PD participants. Response underinformativeness was associated with decreased executive control, mental status, and speech act comprehension measures. However, both speech act priming and utterance informativeness were strongly related to a measure of executive control, and when this variable (i.e., Stroop performance) was controlled, the correlation between speech act priming and utterance underinformativeness was no longer significant. It appears, then, that executive control deficits are related to the ability to comprehend and produce conversational utterances.
\end{abstract}

Keywords: Pragmatic Deficits; Parkinson's Disease; Conversation Maxims

\section{INTRODUCTION}

A fundamental skill for successful language use is the ability to contribute appropriately to a conversation. This is not a trivial skill; it involves monitoring the overall tenor and direction of a conversation as well as the specific remarks of one's interlocutors. Failing to quickly understand the intended meaning of others' utterances will compromise one's ability to contribute appropriately to a conversation. In this research, we examined the possibility that people with Parkinson's disease (PD) may be impaired in their ability to contribute appropriately to a conversation and that there are several cognitive variables that underlie this capacity.

Although PD is primarily associated with motor dysfunction, it also affects thinking, reasoning, planning and language functions. In fact, Aarsland and colleagues estimate that twenty to thirty percent of nondemented and early-stage PD patients have mild cognitive impairment [1]. McKinlay, Grace, Dalrymple-Alford, and Roger add that cognitive dysfunction in PD includes difficulties with planning, set-shifting, working memory, verbal fluency, and inhibition or cognitive control [2].

Recent research has examined the impact of cognitive impairment on social skills [3]. Results suggest that PD patients with no evidence of cognitive deficits judged social situations to be more awkward than non-PD participants, even when controlling for anxiety. Results also suggest that PD patients diagnosed with mild cognitive impairment (MCI) experienced difficulty categorizing others' actions and sarcastic remarks. Anderson and colleagues state that this pragmatic difficulty may only occur in the context of cognitive impairment because PD participants without MCI and age-matched controls did not demonstrate this effect [3].

\subsection{Parkinson's Disease and Language Use}

In terms of language, patients with PD often exhibit fluency and motor speech disorders, word-finding difficulties, and grammatical difficulties. For example, they tend to use simplified sentence structures with relatively more silent hesitations and pauses at critical sites in a sentence $[4,5]$. In addition, many patients with PD exhibit difficulty in generating words to a target stimulus [6], an effect that is particularly pronounced for verbs $[7,8]$. Patients with PD also tend to produce a smaller number of grammatical utterances than healthy individuals $[9,10]$, and tend to use a larger number of words to describe similar themes [11]. In addition to language production, patients with PD also often exhibit mild to moderate sentence comprehension deficits [12-15].

More recent evidence has emerged documenting the existence of certain pragmatic deficits in PD. For example, Hall, Ouyang, Lonnquist, and Newcombe investigated various pragmatic skills in people with PD and the relationship between those skills and various measures of motor and cognitive dysfunction [16]. PD participants scored significantly lower on a pragmatic function scale than did matched controls, with PD patients demonstrateing deficits in eye contact, intonation, turn taking, re- 
sponse length, and conversation initiation. Other researchers have reported "high-level" language dysfunction in PD including a significant inferencing deficit (i.e., drawing appropriate inferences from short narratives about social interactions) [17] and a disadvantage interpreting figurative language [18] including verbal irony [19].

More recently, Holtgraves and McNamara documented a speech act recognition deficit in people with PD [20]. Speech acts are the specific intention (e.g., compliment, criticize, thank, etc.) that a speaker intends to convey with an utterance. Participants in this study read short scenarios describing interactions between two or more people and then immediately performed a lexical decision task (participants decided whether a target string of letters was a word). Consistent with past research [21, $22]$, non-PD participants performed this task more quickly when the target word named the speech act associated with the preceding utterance, hence demonstrating automatic speech act priming. In contrast, people with PD did not demonstrate this effect, suggesting that speech act priming is slowed or is not an automatic component of comprehension for people with PD. In a second study, participants were given unlimited time to indicate their recognition of the speech act performed with an utterance. $P D$ participants performed signifycantly more poorly on this task than non-PD participants.

The purpose of the present research was to examine possible pragmatic production deficits in PD using a spontaneous language task, and whether such deficits are related to our previously demonstrated pragmatic comprehension deficits [20]. Specifically, we examined the extent to which a person's utterances abide by Grice's quantity maxim (i.e., make your utterance as informative as required) [23]. If people with PD tend not to recognize the language-based intention of their interlocutor, as demonstrated by Holtgraves and McNamara [20], then their contributions to a verbal exchange should tend to be relatively under-informative. We examined the informativeness of the utterances of people with and without PD and the relationship between utterance informativeness and measures of speech act priming, speech act recognition, executive control, mental status, and the severity of motor dysfunction. In general, we expected PD participants to be more under-informative than non-PD participants, and that the degree of under-informativeness would be negatively correlated with the degree of automatic speech act priming performance, mental status, and executive control and positively correlated with degree of motor severity (for PD participants).

\section{METHOD}

\subsection{Participants}

Participants were 20 (2 females) individuals diagnosed with idiopathic Parkinson's disease (mean age $=64.7$, mean Hoehn \& Yahr stage $=2.0)$, and 20 (8 females) non-PD individuals (mean age $=54.4$ ). PD participants were recruited from the Boston University School of Medicine/Boston Medical Center Movement Disorder Clinics and the Boston VA Parkinson/Movement Disorder Clinics. Non-PD participants were recruited via advertisement in Boston area media outlets and via the Harvard Consortium for Aging Studies. All non-PD participants reported histories of chronic illness not typically associated with cognitive dysfunction. Most (80\%) reported histories of chronic low back pain. The rest reported histories of other chronic ailments like diabetes, headaches and migraine. This type of control group is ideal because it allows us to draw conclusions about pragmatic language deficits specifically associated with $\mathrm{PD}$, rather than general illness. There was no difference in education level between the PD participants (14.4 years) and the non-PD participants (14.9 years), $t(38)=$ $0.58, p>0.5$. However, relative to the PD participants, the non-PD group had more females $(40.0 \%$ vs. $10.0 \%$, $\left.\chi^{2}=4.8, p<0.05\right)$, and was younger (54.4 vs. $64.7, t(37)$ $=-2.91, p<0.01)$. Subsequent analyses indicated that none of these variables were significantly correlated with the dependent measure of interest (all $p s>0.15$ ).

PD participants' diagnosis was agreed upon by a specialist in PD and at least one other neurologist. None of the participants met DSM-IV criteria for dementia. All PD participants were currently taking dopaminergic medication with optimal effects (i.e., motor symptoms were controlled). All patients were required to have had at least one CT or MRI scan during their illness to rule out history of brain injury. Other exclusion criteria included: patients with Parkinsonism from known causes (e.g., encephalitis), degenerative diseases mimicking PD (e.g., striatonigral degeneration), an abnormal CT or MRI scan showing basal ganglia atrophy or calcification and or stroke, an inability to obtain informed consent from patient due to an incapacity on the part of patient to understand purpose and risks of study, a history of ongoing alcohol or drug abuse, a history of psychiatric or psychotic disorder, and patients currently on anti-depressant or anti-psychotic medications as these medications may influence communication functions.

\subsection{Procedure}

Participants provided demographic and medical information at the start of the study and then completed the following tasks over a three-day period (two-three hours per day).

Language Production Measure. Interviewers conducted semi-structured interviews with each participant individually. The interviews consisted of questions regarding the interviewees' family, work history, daily ac- 
tivities, etc. Interviews lasted approximately fifteen minutes. All interviews were recorded and subsequently transcribed for analyses. Two coders who were blind to participants' group membership independently coded participants' individual interview responses. Coders developed a five-point coding scale for informativeness by examining a set of interviews from a different data set and then collapsed the scale to a three-point scale (under-informative; i.e., too little information provided; over-informative; i.e., too much information provided; appropriate level of information). The coders used this scale to code each turn in the conversation for level of informativeness. Tangential information that did not answer the interviewer's questions was coded as overinformative.

Speech Act Comprehension Measures. We used the previously reported speech act comprehension data as described in Holtgraves and McNamara [20]. In that research participants completed a speech act priming task in order to assess their ability to automatically recognize a speaker's intention. Participants read forty-eight brief scenarios describing an interaction between two people. Each scenario ended in a remark from one of the characters. Participants then performed a lexical decision task. On one-half of the critical trials the to-be-judged target named the speech act performed with the prior utterance; on the remaining critical trials, the target did not name the speech act performed with the prior utterance. Participants' reaction times to the probes were recorded and constituted the speech act priming measure. In a second, untimed task, participants read similar materials and were simply asked to identify which speech act a speaker had performed (speech act recognition measure). A detailed description of these two tasks and the corresponding results are presented in Holtgraves and McNamara [20].

Cognitive Measures. We used the previously reported cognitive data as described in Holtgraves and McNamara [20]. All participants completed the Mini-Mental State Examination (MMSE), a brief measure developed to assess general cognitive functioning [24]. Participants also completed the Stroop color-word interference task, a measure of cognitive control [25]. Although there is no standard measure of cognitive control, this version of the Stroop task involves both planning and inhibition and is a reasonable measure of cognitive control for the purposes of this study. In this version of the Stroop task, participants were required to name the color of patches of ink on Card 1 and read the color words printed in black ink on Card 2. On Card 3, the first interference card, color words were printed in colors incongruent with the word represented, and participants were asked to name the color of the ink the word was printed in for each item. On Card 4, the second interference card, color words were also printed in ink colors incongruent with the words represented. However, half of the items were surrounded by a box. Participants were instructed to read the word if it was placed in a box, but name the color of the ink when the word was not surrounded by a box. Participants' overall Stroop scores were calculated as the total time taken to complete Card 1 (name the colors) minus the time taken to complete Card 4.

Motor Severity Measure. Motor symptom severity was assessed using a modified Unified Parkinson's Disease Rating Scale (UPDRS), a standard assessment of motor symptom severity that demonstrates acceptable interrater reliability [26]. Only the UPDRS motor severity scale score was used for analyses. This scale also assesses activities of daily living (ADLs) and potential motor effects of levodopa treatment (i.e. dyskinesia, autonomic signs, etc.).

\section{RESULTS}

Overall agreement rate between the two interview coders was $78.5 \%$, and all disagreements were resolved through discussion. Consensus coding decisions following reliability checks were used for subsequent statistical analyses. The dependent measure used in the following analyses was the percentage of responses that were under-informative. There were no significant group differences for the over-informative and appropriate levels of informativeness categories (all $p s>0.20$ ), and none of the other variables examined in this research (Stroop, speech act priming performance) correlated significantly with the over and appropriately informative categories (all $p s>0.20$ ). Mean scores for the PD and non-PD groups on all reported measures are displayed in Table $\mathbf{1 .}$ The correlations between all reported measures are displayed in Table 2.

Overall, the percentage of utterances classified as under-informative was relatively small $(M=3.23 \% ; S D=$ 0.07). However, and as expected, a greater number of under-informative utterances were produced by PD participants $(M=4.3 \%)$ than were produced by the nonimpaired participants $(M=1.1 \%), F(1,38)=3.815, p=$ 0.058 .

As reported in Holtgraves and McNamara [20], there was a significant Group (PD vs. non-PD) X Target Type (Speech Act vs. non-Speech Act) interaction for lexical decision speed, $F(1,38)=5.21, p<0.04$. The non-PD participants demonstrated automatic speech activation (i.e., had significantly faster lexical decision speeds for targets naming the speech act performed with the prior utterances (1182 ms) relative to the control utterances (1327 ms)), but PD participants did not (1990 ms vs. $1873 \mathrm{~ms})$. In addition, when given unlimited time, PD participants identified significantly fewer speech acts $(35 \%)$ than did the non-PD participants $(42 \%)$. However, 
Table 1. Correlation matrix for all reported measures.

\begin{tabular}{lccccc}
\hline \multirow{2}{*}{ Measures } & \multicolumn{3}{c}{ Measures } \\
\cline { 2 - 6 } & Underinformativeness & Priming & Recognition & Mental Status & Stroop \\
\hline Under-informativeness & 1.00 & $-0.34^{\mathrm{a}}$ & -0.25 & $-0.45^{\mathrm{b}}$ & $0.42^{\mathrm{b}}$ \\
Priming & & 1.00 & 0.20 & 0.29 & $-0.74^{\mathrm{b}}$ \\
Recognition & & 1.00 & 0.06 & -0.30 \\
Mental Status & & & 1.00 & -0.28 \\
Stroop & & & & 1.00 \\
\hline
\end{tabular}

${ }^{\mathrm{a}} p<0.05 ;{ }^{\mathrm{b}} p<0.01$.

Table 2. Group comparisons for all reported measures.

\begin{tabular}{lcccc}
\hline \multirow{2}{*}{ Comparison Variables } & \multicolumn{3}{c}{ Means and Statistical Comparisons } \\
\cline { 2 - 4 } Age & $\mathrm{PD}(N=20)$ & Non-PD $(N=20)$ & $t$ & $p$ \\
Education & 64.65 & 54.35 & -2.91 & 0.01 \\
Percent of under-informative utterances & 14.40 & 14.93 & 0.58 & 0.57 \\
UPDRS & $4.30 \%$ & $1.10 \%$ & 1.95 & 0.06 \\
MMSE & 27.15 & & & 0.00 \\
Stroop & 27.06 & 28.32 & 3.24 & 0.06 \\
Priming & 83.30 & 46.90 & -1.91 & 0.03 \\
Recognition & -117.1 & 145.45 & 2.28 & 0.21 \\
\hline
\end{tabular}

in this restricted analysis this difference was not significant, $F(1,38)=1.62, p=0.21^{1}$

Correlations between the under-informativeness measure and speech act priming, speech act recognition, mental status, and executive control were computed. The degree of under-informativeness was negatively correlated with performance on the speech act priming task, $r(38)=-0.336, p=0.034$, indicating that participants who were poorer at automatically recognizing speech acts tended to be more under-informative in the interview. However, this correlation was marginally larger $(p$ $<0.06$, one-tailed) for the PD participants $(r=-0.33)$ than for the non-PD participants $(r=0.20)$, suggesting that speech act comprehension deficits may be related to the under-informativeness of PD participants more so than for non-PD participants. The correlation between under-informativeness and speech act recognition was also negative but not significant $(r=-0.25, p<0.12)$. However, like speech act priming, this correlation was larger (though not significantly) for the PD participants

\footnotetext{
${ }^{1}$ The ns and values reported here are slightly different from those reported in Holtgraves and McNamara (2010) because the present results are restricted to participants who completed both the interview and the speech act recognition tasks.
}

$(r=-0.27)$ than for the nonimpaired participants $(r=$ $0.12)$.

Under-informativeness was also negatively correlated with performance on the mental status exam, $r(38)$ $=-0.446, p=0.004$, indicating that greater cognitive impairment was associated with more under-informative interview responses, and positively correlated with interference scores on the Stroop task, $r(38)=0.418, p=$ 0.007 , indicating that decreased executive control was associated with more under-informative responses.

In terms of unique variance, partial correlations indicated that the under-informativeness - speech act priming correlation remained marginally significant when controlling for mental status $(r=-0.242, p<0.07)$ but was nonsignificant when controlling for Stroop performance $(r=-0.04, p>0.05)$. Hence, in this research it appears that decrements in executive control may disrupt both automatic speech act activation and conversational appropriateness.

Lastly, under-informativeness was positively correlated with the motor severity score on the UPDRS for the PD participants, $r(18)=0.444, p=0.05$. Hence, greater motor symptom severity was associated with greater under-informativeness for PD participants. 


\section{DISCUSSION}

Prior research has demonstrated certain pragmatic language deficits in people with PD [16-20]. The present results extend this line of research in several ways. First, we demonstrate a specific, previously undocumented, language production deficit in PD. Specifically, PD participants in this study produced marginally significantly more utterances classified as underinformative than did the non-PD participants, a deficit that is consistent with prior research demonstrating reduced information content in the language of people with PD [27]. Second, we demonstrate this deficit during a naturally occurring verbal interaction rather than with a laboratory task. Third, for the PD participants, this deficit was negatively correlated with the ability to automatically recognize others' speech acts. Hence, the ability to recognize a speaker's language-based intention underlies this production deficit. Of course this finding makes intuitive sense; it is difficult to generate meaningful contributions to a conversation without understanding your interlocutors. Fourth, both speech act priming and utterance informativeness were strongly related to a measure of executive control, and when this variable (i.e., Stroop performance) was controlled, the correlation between speech act priming and utterance under-informativeness was no longer significant. It appears, then, that executive control deficits are related to the ability to both comprehend and produce conversational utterances.

This research has several limitations that should be noted. First, our sample was predominately male, an imbalance that was larger for the PD group than for the non-PD group. However, this imbalance for PD participants is consistent with past research which suggests the actual incidence of Parkinson's disease is much higher for men than for women [28]. Although we found no evidence for gender effects (neither main effects nor interactions) in this research, our results may need to be viewed with some caution due to this imbalance. Second, our PD participants were significantly younger than our non-PD participants. Age, however, was not significantly correlated with any of the measures reported in this research. Third, our measure of utterance informativeness was not without some limitations. Although coders were able to achieve an acceptable agreement level using the coding scheme they developed and resolve their disagreements through discussion, this judgment was made by observers rather than conversationalists. The gold standard for utterance informativeness would be the judgments of people participating in the interaction.

The results of this research are important clinically. When working with people with PD, it is important that clinicians understand their tendency to be under-informative in conversation. Clinicians should be patient and ask for additional information when needed. Clinicians should also consider asking for information from other sources (i.e. family members, previous clinicians, etc.), as they may be able to provide information PD patients have difficulty verbalizing.

\section{ACKNOWLEDGEMENTS}

This research was supported by a grant from the NIDCD: 'Pragmatic Language Skills in Patients with Parkinson's Disease', 1R01DC007956-01A2.

\section{REFERENCES}

[1] Aarsland, D., Marsh, L. and Schrag, A. (2009) Neuropsychiatric symptoms in Parkinson's disease. Movement Disorders, 24, 2175-2186. doi:10.1002/mds.22589

[2] McKinlay, A., Grace, R. C., Dalrymple-Alford, J. C. and Roger, D. (2010) Characteristics of executive function impairment in Parkinson's disease patients without dementia. Journal of the International Neuropsychological Society, 16, 269-277. doi:10.1017/S1355617709991299

[3] Anderson, R.J., Simpson, A.C., Channon, S., Samuel, M. and Brown, R.G. (2012) Social problem solving, social cognition, and mild cognitive impairment in Parkinson's disease. Behavioral Neuroscience, Advance online publiccation. doi: $10.1037 / \mathrm{a} 0030250$

[4] Troche, M.S. and Altmann, L.P. (2012) Sentence production in Parkinson disease: Effects of conceptual and task complexity. Applied Psycholinguistics, 33, 225-251. doi:10.1017/S0142716411000336

[5] Illes, J., Metter, E.J., Hanson, W.R. and Iritani, S.S. (1988) Language production in Parkinson's disease: Acoustic and linguistic considerations. Brain and Language, 33, 146160. doi:10.1016/0093-934X(88)90059-4

[6] McNamara, P., Obler, L.K., Au, R., Durso, R. and Albert, M. (1992) Speech monitoring skills in Alzheimer's disease, Parkinson's disease, and normal aging. Brain and Language, 42, 38-51. doi:10.1016/0093-934X(92)90055-J

[7] Bertella, L., Albani, G.G., Greco, E.E., Priano, L.L., Mauro, A.A., Marchi, S.S. and Semenza, C.C. (2002) Noun verb dissociation in Parkinson's disease. Brain and Cognition, 48, 277-280.

[8] Peran, P., Rascol, O., Demonet, J., Celsis, P., Nespoulous, J., Dubois, B., et al. (2003) Deficit of verb generation in nondemented patients with Parkinson's disease. Movement Disorders, 18, 150-156. doi:10.1002/mds.10306

[9] Murray, L.L. (2000) Spoken language production in Huntington's and Parkinson's diseases. Journal of Speech, Language, and Hearing Research, 43, 1350-1366.

[10] Walsh, B. and Smith, A. (2011) Linguistic complexity, speech production, and comprehension in Parkinson's disease: Behavioral and physiological indices. Journal of Speech, Language, and Hearing Research, 54, 787-802. doi:10.1044/1092-4388(2010/09-0085)

[11] Obler, L., Mildworf, B. and Albert, M. (1977) Writing style in the elderly. Academy of Aphasia Abstracts, Montreal. 
[12] Lieberman, P., Friedman, J. and Feldman, L.S. (1990) Syntax comprehension deficits in Parkinson's disease. Journal of Nervous and Mental Disease, 178, 360-365. doi:10.1097/00005053-199006000-00003

[13] Grossman, M., Kalmanson, J., Bernhardt, N., Morris, J., Stern, M.B. and Hurtig, H.I. (2000) Cognitive resource limitations during sentence comprehension in Parkinson's disease. Brain and Language, 73, 1-16.

[14] McNamara, P., Krueger, M., O'Quin, K., Clark, J. and Durso, R. (1996) Grammaticality judgments and sentence comprehension in Parkinson's disease: A comparison with Broca's aphasia. International Journal of Neuroscience, 86, 151-166. doi:10.3109/00207459608986706

[15] Kemmerer, D. (1999) Impaired comprehension of raising-to-subject constructions in Parkinson's disease. Brain and Language, 66, 311-328. doi:10.1006/brln.1999.2022

[16] Hall, D., Ouyang, B., Lonnquist, E. and Newcombe, J. (2011) Pragmatic communication is impaired in Parkinson disease. International Journal of Neuroscience, 121, 254-256. doi:10.3109/00207454.2010.550389

[17] Berg, E., Björnram, C., Hartelius, L., Laakso, K. and Johnels, B. (2003) High-level language difficulties in Parkinson's disease. Clinical Linguistics \& Phonetics, 17, 6380. doi:10.1080/0269920021000055540

[18] Lewis, F.M., LaPointe, L.L., Murdoch, B.E. and Chenery, H.J. (1998) Language impairment in Parkinson's disease. Aphasiology, 12, 193-206. doi:10.1080/02687039808249446

[19] Monetta, L., Grindrod, C.M. and Pell, M.D. (2009) Irony comprehension and theory of mind deficits in patients with Parkinson's disease. Cortex: A Journal Devoted to the Study of the Nervous System and Behavior, 45, 972981. doi:10.1016/j.cortex.2009.02.021

[20] Holtgraves, T. and McNamara, P. (2010) Pragmatic com- prehension deficit in Parkinson's disease. Journal of Clinical and Experimental Neuropsychology, 32, 388-397. doi:10.1080/13803390903130729

[21] Holtgraves, T. (2008) Automatic intention recognition in conversation processing. Journal of Memory and Language, 58, 627-645. doi:10.1016/j.jml.2007.06.001

[22] Holtgraves, T. and Ashley, A. (2001) Comprehending illocutionary force. Memory \& Cognition, 29, 83-90. doi:10.3758/BF03195743

[23] Grice, H.P. (1975) Logic and conversation. In: P. Cole and J. Morgan, Eds., Syntax and semantics, Academic Press, New York, 41-58.

[24] Folstein, M.F., Folstein, S.E. and McHugh, P.R. (1975) Mini-Mental State. A practical method for grading the cognitive state of patients for the clinician. Journal of Psychiatric Research, 12, 189-198. doi:10.1016/0022-3956(75)90026-6

[25] Delis, D. C., Kaplan, E. and Kramer, J.H. (2001) The Delis/Kaplan Executive Function System (D-KEFS). The Psychological Corporation, San Antonio.

[26] Martínez-Martín, P., Gil-Nagel, A., Gracia, L.M., Gómez, J.B., Martínez-Sarriés, J. and Bermejo, F. (1994) Unified Parkinson's disease rating scale characteristics and structure. Movement Disorders, 9, 76-83.

[27] Altmann, L.J.P. and Troche, M.S. (2011) High-level language production in Parkinson's disease: A review. Parkinson's Disease, 2011, 238956. doi: $10.4061 / 2011 / 238956$

[28] Van Den Eeden, S.K., Tanner, C.M., Bernstein, A.L., Fross, R.D., Leimpeter, A., Bloch, D. and Nelson, L.M. (2003) Incidence of Parkinson's disease: Variation by age, gender, and race/ethnicity. American Journal of Epidemiolog, 157, 1015-1022. doi:10.1093/aje/kwg068 